\title{
Integrated photonic circuit in silicon on insulator for Fourier domain optical coherence tomography
}

\author{
Günay Yurtsever ${ }^{*}$,, Pieter Dumon ${ }^{\mathrm{a}}$, Wim Bogaerts ${ }^{\mathrm{a}}$, Roel Baets ${ }^{\mathrm{a}}$ \\ ${ }^{a}$ Ghent University - IMEC, Photonics Research Group, Department of Information Technology \\ (INTEC), Sint-Pietersnieuwstraat 41, 9000 Gent, Belgium
}

\begin{abstract}
Optical coherence tomography (OCT) is a medical imaging technology capable of producing high-resolution, crosssectional images through inhomogeneous samples, such as biological tissue. It has been widely adopted in clinical ophthalmology and a number of other clinical applications are in active research. Other applications of OCT include material characterization and non-destructive testing. In addition to current uses, OCT has a potential for a much wider range of applications and further commercialization. One of the reasons for slow penetration of OCT in clinical and industrial use is probably the cost and the size of the current systems. Current commercial and research OCT systems are fiber/free space optics based. Although fiber and micro-optical components have made these systems portable, further significant miniaturization and cost reduction could be achieved through the use of integrated photonic components. We demonstrate a Michelson interferometer using integrated photonic waveguides on nanophotonic silicon on insulator platform. The size of the interferometer is $1500 \mu \mathrm{m}$ x $50 \mu \mathrm{m}$. The structure has been tested using a mirror as a reflector. We can achieve $40 \mu \mathrm{m}$ axial resolution and $25 \mathrm{~dB}$ sensitivity which can be substantially improved.
\end{abstract}

Keywords: optical coherence tomography, silicon photonics, integrated photonics, silicon on insulator

\section{INTRODUCTION}

During the last decade there has been a significant advancement in integrated silicon photonics, specifically due to a need for optical interconnects in multi-core processors. Passive structures like low-loss waveguides, spot size converters, polarization splitters, demultiplexers, resonators and active components such as modulators, switches, and heterogeneously integrated photodetectors and lasers have been demonstrated on silicon ${ }^{1}$. Nanophotonic silicon on insulator (SOI) is a versatile platform for a variety of densely integrated silicon photonic components that has been used by many research groups. An SOI wafer consist of a thin top $\mathrm{Si}$ (refractive index, $\mathrm{n}=3.45$ ) layer sitting on silica $\left(\mathrm{SiO}_{2}\right.$, $n=1.45$ ) layer, which is carried on a thick Si substrate. Photonic components are realized by etching the top Si layer, resulting in high refractive index contrast in all directions. Using wafer scale CMOS compatible processes, low loss $(<2.4 \mathrm{~dB} / \mathrm{cm})$ waveguides with core sizes of $0.1 \mu \mathrm{m}^{2}$ and bend radii of $5 \mu \mathrm{m}$ can be realized. Using such wafer scale processes for silicon, low-cost, high density, integrated photonic components can be mass-fabricated and integrated with CMOS electronics $^{2}$. Due to absorption of the silicon in the visible range, the components are designed for wavelengths above $1100 \mathrm{~nm}$. Although silicon is not an efficient light emitter and detector in the near infrared wavelengths, active elements can be heterogeneously integrated on top of the silicon wafer ${ }^{3}$. Using the SOI platform together with heterogeneous integration of sources and detectors, significant part of an optical coherence tomography system can be realized on chip in the near future.

Optical Coherence Tomography (OCT) can benefit from the progress in integrated photonics for miniaturization and cost reduction. However, there has been little research on utilizing integrated photonics for OCT. Culernann et al. demonstrated a passive photonic circuit for OCT fabricated on silica. The device consisted of cascaded splitters and combiners ${ }^{4}$. The photonic structures in glass are very low loss, transparent in the visible, however, due large bend radii, the size of the components are almost two orders of larger than the ones on silicon. In another study, Margallo-Baas et al. demonstrated an integrated photonic structure on silicon on insulator (SOI) for time domain OCT, where the delay line

" gunay@intec.ugent.be; phone: +32 9264 8930; fax: +32 9264 3593; http://photonics.intec.ugent.be 
was implemented with thermal tuning of a waveguide in the reference $\mathrm{arm}^{5}$. In this paper, we present an integrated Michelson interferometer for Fourier domain OCT fabricated on SOI using CMOS compatible fabrication process.

\section{DESIGN}

An illustration of the integrated Michelson interferometer is given in Figure 1. Fiber coupled tunable laser is coupled to the planar photonic chip via a grating coupler which has a FWHM bandwidth of $56 \mathrm{~nm}$ and $25 \%$ coupling efficiency. The size of the grating coupler is $10 \mu \mathrm{m}$ and is tapered to a single mode, polarization maintaining waveguide. The waveguides are etched $220 \mathrm{~nm}$ deep and $450 \mathrm{~nm}$ wide with deep UV lithography. Then the light is split into reference

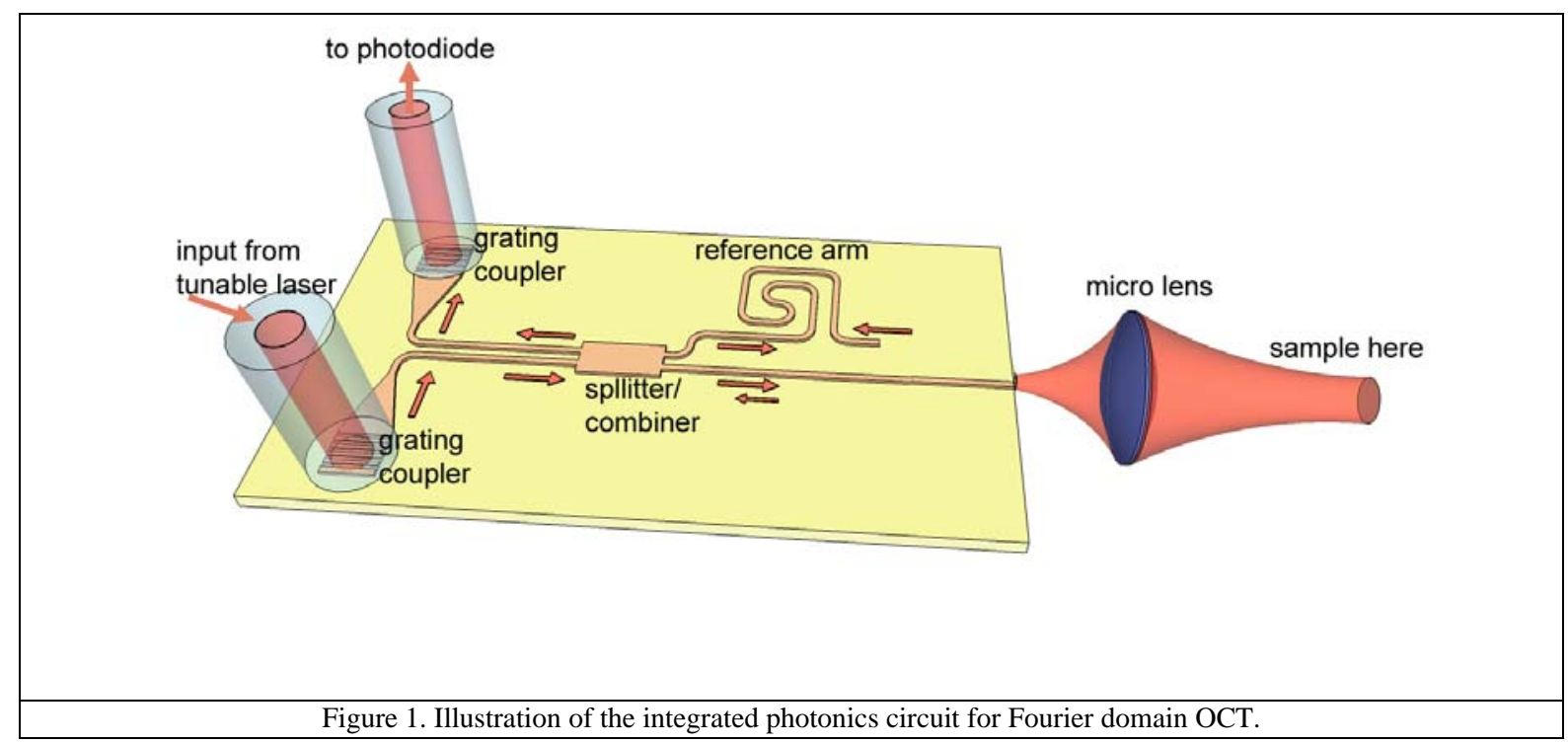

and sample arms with a 50/50 broadband (bandwidth >100nm) multimode interference splitter/combiner. The insertion loss of the splitter/combiner is less than $1 \mathrm{~dB}$. The reference arm consists of a $9 \mathrm{~mm}$ long spiral waveguide with $5 \mu \mathrm{m}$ bend radii and ends with a silicon air interface. The flat waveguide end acts as a broadband reflector with $30 \%$ reflection efficiency. In the sample arm, the light is coupled out of the chip from a waveguide facet. The output from the waveguide facet is imaged onto the sample with a micro lens. The reflections from the sample and reference arms are combined with the splitter/combiner and coupled to a fiber which guides the light to a photodiode. The sample is coated with $1 \mu \mathrm{m}$ thick benzocyclobutene $(\mathrm{BCB}$, refractive index $\mathrm{n}=1.54)$ to reduce the group velocity dispersion of the waveguides.

\section{FABRICATION}

The device is fabricated on a $200 \mathrm{~mm}$ diameter SOI wafer with $220 \mathrm{~nm}$ thick Si top layer on top of $2 \mu \mathrm{m}$ thick $\mathrm{SiO}_{2}$ using 193nm deep-UV lithography in combination with inductively coupled plasma reactive ion etching. The fabrication was done through ePIXfab silicon photonics platform (www.epixfab.eu ) at IMEC nanoelectronics and nanotechnology research center, Belgium. The sample is cleaved to create the facet at the end of the sample arm to couple the light out of the chip. A light microscope image of the fabricated structure is given in Figure 2. The size of the Michelson interferometer is only 1500 x $50 \mu \mathrm{m}$. In a wafer scale process, hundreds of these structures can be fabricated in a single run. In addition to advantages of cost and size, miniaturization of OCT through integrated photonics components will also provide more stable polarization maintaining interferometers compared to fiber 
based systems.

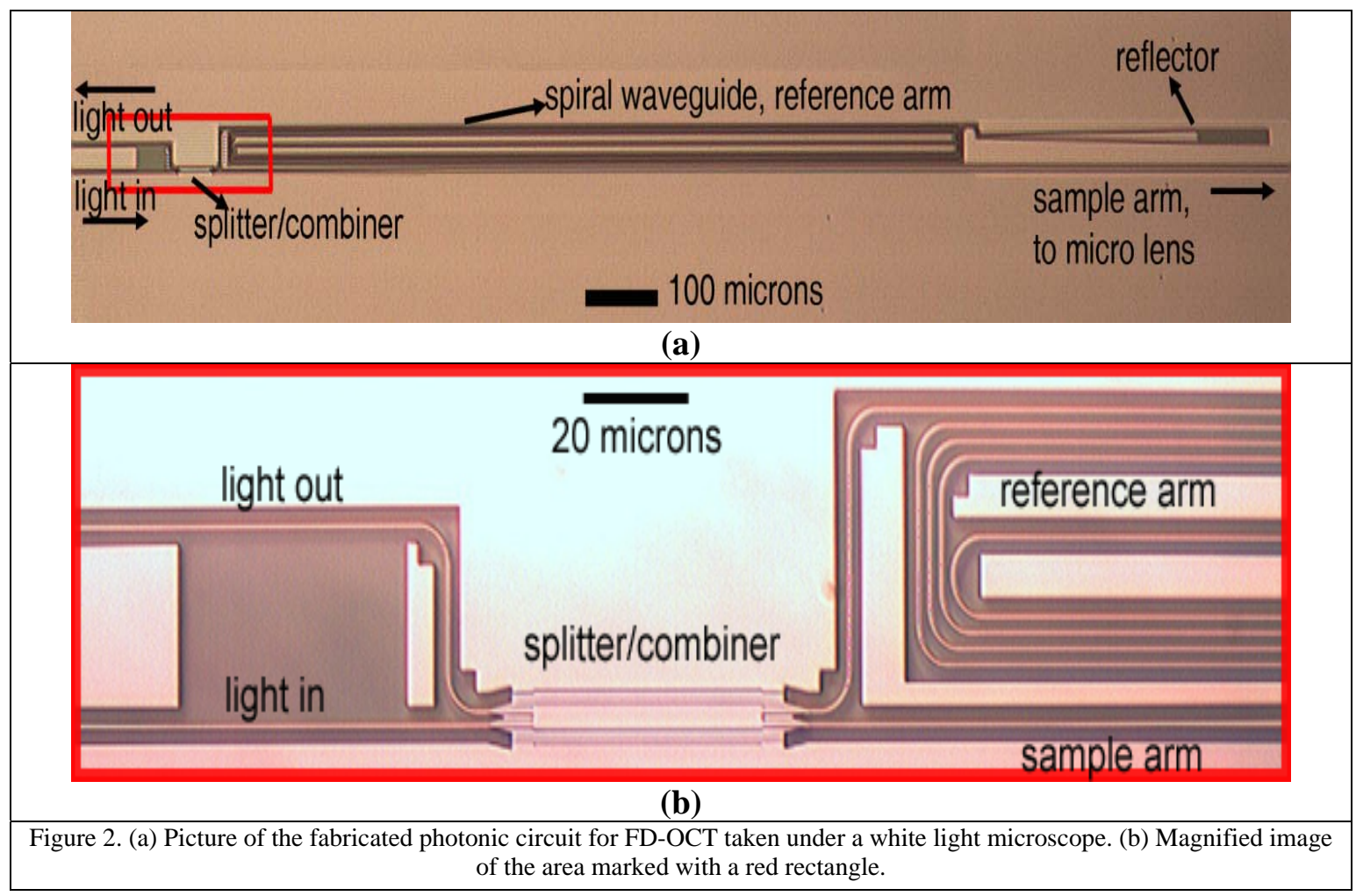

\section{MEASUREMENT}

The measurement setup is given in Figure 3. The chip is placed on vacuum holder. A single mode fiber from a tunable laser (1510 - $1610 \mathrm{~nm}, 1 \mathrm{~Hz}$ tuning frequency) couples the light to the chip. A micro lens with an NA of 0.53

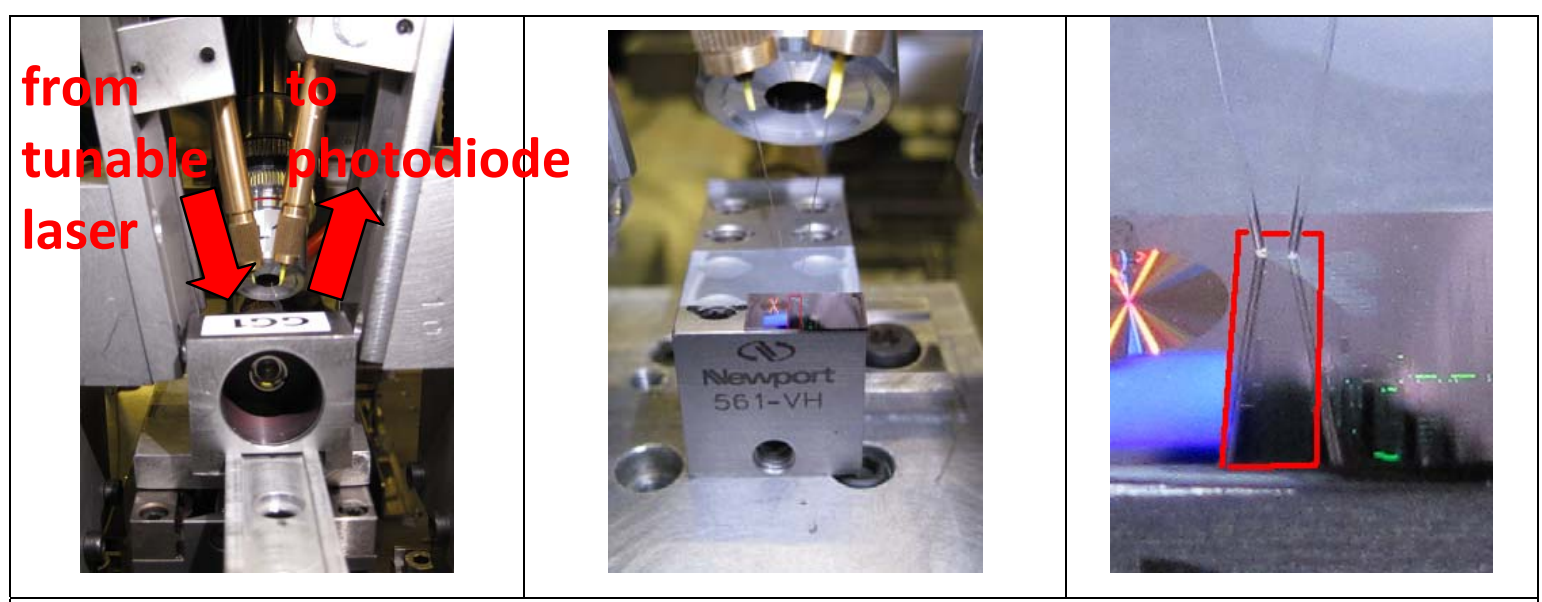

Figure 3. a. The measurement setup b. The measurement setup without the micro lens c. Close up of the measurement setup, the structure is located in the red rectangle, input and output fibers are clearly shown. 
is used to collimate the light and send onto a gold mirror. The interference of the reference arm and sample arm are coupled out of the chip with a grating coupler to a fiber and send to a photodiode. The OCT signal from the mirror is given in Figure 4. The axial resolution is around $40 \mu \mathrm{m}$, which is higher than theoretical $27 \mu \mathrm{m}$ in air for $40 \mathrm{~nm}$ FWHM bandwidth and no group velocity dispersion (GVD). From fully vectorial simulations with Fimmwave mode solver (Photon Design, Inc), the group velocity dispersion of the fundamental TE mode of a $220 \mathrm{~nm} x 450 \mathrm{~nm}$ waveguide with BCB cladding is found to have a GVD of $-250 \mathrm{fs}^{2} / \mathrm{cm}$. The GVD of $5 \mu \mathrm{m}$ bends are also calculated to be in that order. The broadening of the axial resolution is probably due to GVD mismatch, which can be reduced by engineering the GVD of the waveguides by changing their dimensions. Flexibility of engineering the GVD of silicon waveguides may also be used to fully compensate the GVD mismatch for tissue imaging,

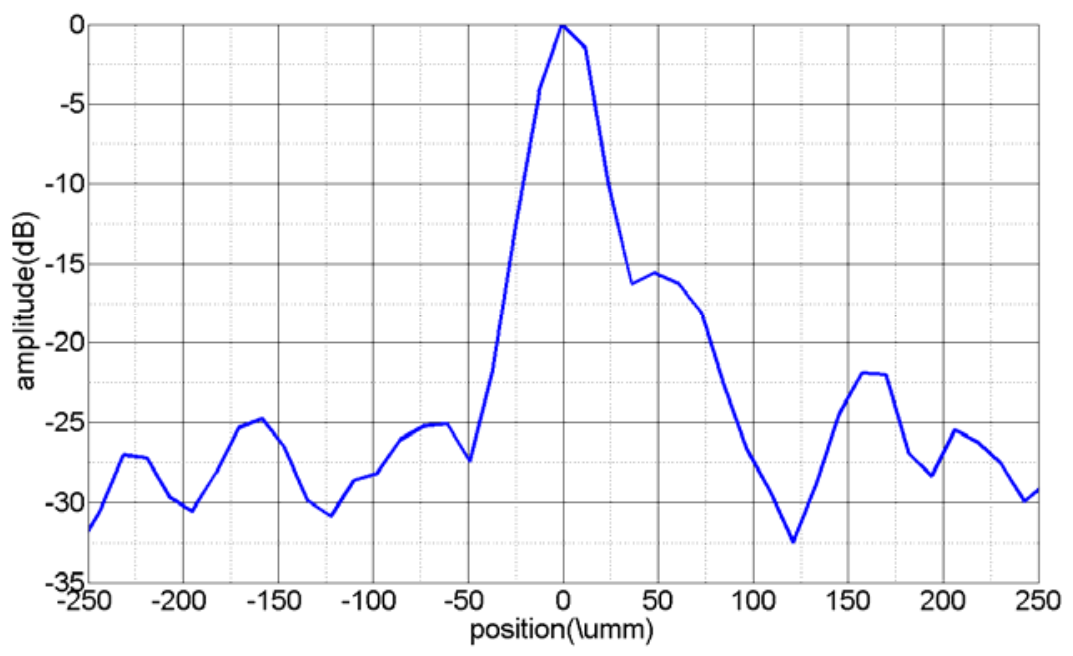

Figure 4. OCT signal from a mirror.

The sensitivity of the system is around $25 \mathrm{~dB}$. One of the main reasons for a low sensitivity is the facet where we have loss of $10 \mathrm{~dB}$ power for each pass, reducing the sensitivity by $40 \mathrm{~dB}$. The facet was defined by cleaving the sample and is not polished. The efficiency of the grating couplers also reduces the sensitivity by $24 \mathrm{~dB}$. Rather than a facet and grating couplers, a broadband (>100 nm) spot size converter, which has a loss of only $<2 \mathrm{~dB}$ can be used to couple the light in and out of the chip. This would bring the sensitivity to a level where tissue imaging can be possible.

\section{CONCLUSIONS}

In this study, we have demonstrated a Michelson interferometer for OCT with an ultra small footprint. The axial resolution and sensitivity can be easily improved to a level sufficient for in-vivo imaging with small changes in the design. Passive structures for OCT, such as waveguides, splitters/combiners, couplers, tapers, reflectors are the first step towards integrated OCT. Even without inclusion of active structures such as sources, detectors and modulators, OCT can benefit from such compact and robust components. A complete OCT system on a single chip will be also possible in near future with inclusion of active components. 


\section{ACKNOWLEDGEMENTS}

This work is supported by the Institute for the Promotion of Innovation through Science and Technology in Flanders

(IWT-Vlaanderen) and Verhaert Inc. Wim Bogaerts acknowledges the Flemish Fund for Scientific

Research (FWO) for a postdoctoral grant.

\section{REFERENCES}

[1] Daldosso N., Pavesi L., "Nanosilicon photonics," Laser and Photonics Reviews 3(6), 508-534 (2009).

[2] Bogaerts W., Baets R., Dumon P., Wiaux V., Beckx S., Taillaert D., Luyssaert B., Van Campenhout J., Bienstman P., Van Thourhout D., "Nanophotonic waveguides in silicon-on-insulator fabricated with CMOS technology," Journal of Lightwave Technology 23( 1), 401-412 (2005).

[3] Roelkens G., Liu L., Van Thourhout D., Baets R., Notzel R., Raineri F., Sagnes I., Beaudoin G., Raj R., "Light emission and enhanced nonlinearity in nanophotonic waveguide circuits by III-V/SOI heterogeneous integration", Journal of Applied Physics 104(3), 033117 (2008).

[4] Culernann D., Knuettel A., Voges E., "Integrated optical sensor in glass for optical coherencetomography", IEEE Journal of Selected Topics in Quantum Electronics 5(6), 730-734 (2000).

[5] Margallo-Baas E., Pandraud G., French P.J., "Thermo-Optical Delay Line for Miniature Optical Coherence Tomography", in the Proceedings of the Conference on Coherence Domain Optical Methods and Optical Coherence Tomography in Biomedicine XII 6847, S8470 (2008).

[6] Roelkens G., Dumon P., Bogaerts W., Van Thourhout D., Baets R., "Efficient Silicon-on-Insulator fiber coupler fabricated using 248nm deep UV lithography” IEEE Photonics Technology Letters , 17(12), 2613-2615 (2005). 